\section{CAMBIO CLIMÁTICO, PRECIPITACIONES, SOCIEDAD Y DESASTRES EN AMÉRICA LATINA: RELACIONES Y NECESIDADES}

\section{[CLIMATE CHANGE, RAINFALL, SOCIETY AND DISASTERS IN LATIN AMERICA: RELATIONS AND NEEDS]}

\author{
Alfonso J. Rodríguez-Morales ${ }^{1}$
}

Sr. Editor. El comienzo del año 2010 estuvo marcado en muchos países de América Latina por el impacto de una fuerte anomalía climática, cuantificada por la National Oceanographic and Atmospheric Administration (NOAA) de EUA, como un fuerte EI Niño (Strong El Niño), con valores del ONI (Oceanic Niño Index) (promedio de la anomalía de la temperatura de la superficie del mar en la región 3,4 del Niño, en el Océano Pacífico) (1) que oscilaron entre 1,5 y 1,8 para el período noviembre 2009 - marzo 2010, solo comparables con la temporada 1997-1998 (que osciló entre 0,5 y 2,5), y que tuvo reconocidos impactos en América Latina, incluyendo considerables cambios en el patrón de diversas enfermedades transmisibles (1-3). Desde 1997-1998 no se registraba una anomalía climática como la observada en la temporada 20092010.

En muchos países, tal calentamiento tuvo un considerable impacto en las sequías, aumento de los incendios forestales, e incluso en disminución del potencial de producción hidroeléctrica, como ocurrió en Venezuela. Lamentablemente las anomalías que el clima está mostrando se dirigen a ambos extremos, pues durante el mes de julio de 2010 se inició un cambio hacia el enfriamiento y lluvias, con valores de ONI de -1,0 (La Niña), que alcanzaron durante el mes de noviembre de $2010-1,4$, comparable a lo que fue clasificado como una La Niña moderada en la temporada 2007-2008 (con valores del ONI que alcanzaron también -1,4). Todo esto, conllevando a fuertes precipitaciones en diversos países de la región (Figura 1).

Médico tropicalista, PhD (c), Departamento de Medicina Preventiva y Social, Escuela de Medicina Luis Razetti, Facultad de Medicina, Universidad Central de Venezuela, Caracas, Venezuela.

Recibido: 15-12-10 Aprobado: 12-01-11

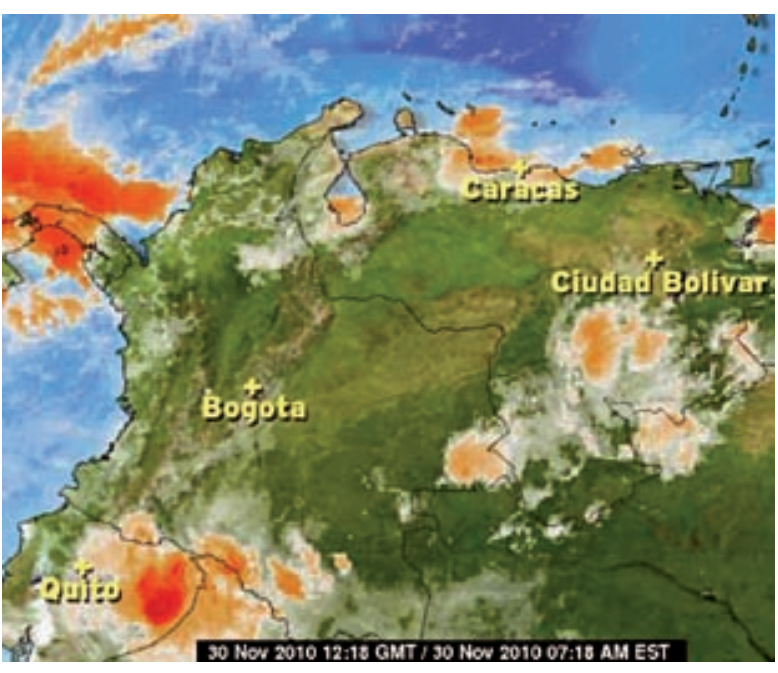

Figura 1. Imagen del satélite GOES del 30 de noviembre de 2010, mostrando fuerte nubosidad sobre la región norte de Venezuela asociada a las precipitaciones más intensas (color naranja).

Todas estas anomalías climáticas han tenido, y seguirán teniendo, considerables impactos en la sociedad, incluyendo una cantidad relevante de efectos en la salud pública ${ }^{1}$. El mes de noviembre de 2010 marcó la caída de fuertes precipitaciones en países como Colombia y Venezuela, que registraron una gran cantidad de eventos adversos o desastres asociados con dichas anomalías meteorológicas, con una considerable proporción de damnificados como consecuencia de inundaciones y deslizamientos que inhabilitaron y destruyeron viviendas (Figura 1).

En Colombia dejó casi 2 millones de afectados y 130 mil en Venezuela (entre noviembre y diciembre de 2010), con 246 muertes en el primer país y 34 en el segundo, hasta el 13 de diciembre de 2010. Por ejemplo, en Venezuela, donde la cifra de damnificados es menor, han tenido que habilitarse 950 albergues en 14 estados del país, 300 de ellos en el Distrito Capital, con las consecuentes implicaciones para la salud pública que ello tiene, por el riesgo de transmisión de agentes causales de diarreas e infecciones respiratorias, entre otras.

Previamente se ha descrito el impacto del cambio climático sobre enfermedades transmisibles y no transmisibles ${ }^{(1-3)}$; sin embargo, mas allá de los efectos directos e indirectos a la salud humana, sus efectos en conllevar a inundaciones pueden ser socialmente devastadores ${ }^{(4)}$. Se considera que las inundaciones son el evento de desastre mas común que puede conllevar a una considerable morbilidad y mortalidad a nivel mundial, con impactos que están en relación directa a la localización y topografía de las áreas afectadas, así como las condiciones demográficas y las características del medio ambiente ${ }^{(4)}$. 
Es por ello que se hace necesario, en el plano de la salud pública, el generar mapas de vulnerabilidad a anomalías climáticas, ya que ellos no existen en la gran mayoría de los países de la región. Por ejemplo, en Venezuela durante las dos semanas de eventos naturales asociados a las intensas precipitaciones (diciembre 2010), las zonas urbanas más afectadas, como era de esperar, son las áreas marginales de ciudades como Caracas, donde las condiciones de las viviendas son precarias e inestables, construidas en cerros y montañas sin los debidos estudios de suelos y la correspondiente colocación de bases que puedan resistir las lluvias.

Estos eventos, tienen consecuencias directas y tempranas en la morbimortalidad de la población afectada, incluida la muerte por la destrucción de viviendas con sus habitantes adentro, así como otras de corto y mediano plazo asociadas con las precipitaciones, como infecciones respiratorias, diarreas, dermatitis y las consecuentemente generadas por hacinamiento en los centros de albergue.

El generar mapas de riesgo a partir de las consecuencias y experiencias encontradas en situaciones como las descritas, puede ayudar a disminuir la vulnerabilidad y aumentar las formas de mitigar sus impactos, al poder tomar medidas antes del inicio de temporadas de lluvias, puesto que pueden ser predecidas por los actuales modelos climatológicos. Recientemente, en algunos países se ha intentado incorporar el uso de tecnologías geoespaciales, no solo en la predicción y el modelaje del comportamiento de enfermedades transmisibles ${ }^{(1-3)}$, sino también incorporar estas herramientas en la comunicación de riesgos a la vida humana en poblaciones de las zonas marginales de las ciudades ${ }^{(5)}$. De allí, que el presente es un llamado de reflexión a incorporar estas herramientas y su aplicación en la salud pública de América Latina con el fin de disminuir el impacto del cambio climático, con sus consecuen- tes efectos como las intensas lluvias, que producen muerte e interrupción de las actividades de la sociedad con el consecuente daño social y económico que estas anomalías del clima pueden causar en países menos preparados para estas situaciones.

\section{Conflictos de Interés}

Los autores declaran no tener conflictos de interés en la publicación de este artículo.

\section{REFERENCIAS BIBLIOGRÁFICAS}

1. Rodriguez-Morales AJ, Risquez A, Echezuria L. Impact of Climate Change on Health and Disease in Latin America. En: Simar S (Editor). Climate Change and Variability. Croatia: Sciyo; 2010. p. 463-486.

2. Cabaniel G, Rada L, Blanco JJ, Rodriguez-Morales AJ, Escalera JP. Impacto de Los Eventos de El Niño Southern Oscillation (ENSO) sobre la Leishmaniosis Cutánea en Sucre, Venezuela, a través del Uso de Información Satelital, 1994 - 2003. Rev Peru Med Exp Salud Publica. 2005;22(1):32-38.

3. Benítez JA, Rodríguez A, Sojo $M$, Lobo $H$, Villegas $C$, Oviedo L, Brown E. Descripción de un Brote Epidémico de Malaria de Altura en un área originalmente sin Malaria del Estado Trujillo, Venezuela. Boletín de Malariología y Salud Ambiental. 2004; 44(2):93-100.

4. Du W, FitzGerald GJ, Clark M, Hou XY. Health impacts of floods. Prehosp Disaster Med 2010;25(3):265-72.

5. Mills JW, Curtis A. Geospatial approaches for disease risk communication in marginalized communities. Prog Community Health Partnersh. 2008;2(1):61-72.

Correspondencia: Prof. Alfonso J. Rodríguez-Morales, MD, MSc, DTM\&H, FFTM RPCS(Glasg)

Dirección: Sección de Inmunoparasitología, Piso 1, Instituto de Medicina Tropical, UCV, Ciudad Universitaria. Caracas 1050, Venezuela.

Correo electrónico: alfonsorm@ula.ve 